\title{
La aplicación de la terapia EMDR en el trastorno límite de la personalidad
}

\author{
Dolores Mosquera \\ Instituto para el estudio del Trauma y los Trastornos de la Personalidad, A Coruña, España
}

Andrew M. Leeds

Sonoma Psychotherapy Training Institute, Santa Rosa, California, EE.UU.

Anabel Gonzalez

Complejo Hospitalario Universitario de A Coruña, A Coruña, España

\begin{abstract}
Existe un interés creciente en usar la terapia de desensibilización y reprocesamiento mediante movimientos oculares (EMDR) más allá del trastorno por estrés postraumático; para el cual dicha aplicación está bien consolidada. Con el fuerte consenso académico de que las experiencias vitales adversas y traumáticas contribuyen al desarrollo del trastorno límite de la personalidad (TLP), parecería que EMDR podría aportar mucho al tratamiento de las personas con TLP. Sin embargo, dadas las características específicas de estos pacientes, la aplicación de la terapia EMDR a su tratamiento puede ser todo un reto y son necesarias pequeñas adaptaciones de los procedimientos estándar de EMDR para el TEPT. Este artículo proporciona una orientación a los principios y estrategias para preparar de manera segura y eficaz a los pacientes con TLP para la terapia EMDR, y para acceder a los orígenes traumáticos del TLP y reprocesarlos. Se ofrecen ejemplos clínicos a lo largo del artículo.
\end{abstract}

Palabras clave: trastorno límite de la personalidad; EMDR; psicoterapia; trauma

$\mathbf{E}$ n el abordaje psicoterapéutico de desensibilización y reprocesamiento mediante movimientos oculares (EMDR), se considera que los problemas psicológicos están causados principalmente por el efecto acumulado de las experiencias adversas y traumáticas no resueltas (Shapiro, 2007). La terapia EMDR ha demostrado ser un tratamiento eficaz, eficiente y bien tolerado para el trastorno de estrés postraumático (TEPT; Bisson y Andrew, 2007; Foa, Keane, Friedman y Cohen, 2009; Ursano et al., 2004) y es efectivo para el TEPT de inicio tanto en la edad adulta como en la infancia (Adler-Tapia y Settle, 2009; Field y Cottrell, 2011; Korn, 2009; van der Kolk et al., 2007). En la terapia EMDR, al paciente se le indica que note de manera plenamente consciente lo que ocurre con las representaciones de las percepciones, emociones, sensaciones, impulsos de acción y autoafirmaciones perturbadoras o disfuncionales, mientras presta atención a una serie de tandas de estimulación bilateral (EBL), que implican movimientos oculares, toques o tonos alternantes (Leeds, 2009; Shapiro, 2001). Gran cantidad de investigaciones apoyan la hipótesis de que el trastorno límite de la personalidad (TLP) está muy relacionado con experiencias vitales adversas y traumáticas en los primeros años de vida (Ball y Links, 2009; Horesh et al., 2008; Tyrka, Wyche, Kelly, Price y Carpenter, 2009; Zanarini et al., 2002); sin embargo, pocos estudios publicados describen la aplicación de EMDR a pacientes con TLP.

Korn y Leeds (2002) aportaron pruebas de un alivio sintomático significativo después de aplicar el desarrollo y la instalación de recursos (DIR) en la fase de estabilización del tratamiento en dos casos de trastorno por estrés extremo no especificado (TEENE/ DESNOS) que también cumplían los criterios para un TLP; sin embargo, no informaron del uso posterior

This article originally appeared as Mosquera, D., Leeds, A., \& Gonzalez, A. (2014) Application of EMDR Therapy for Borderline Personality Disorder. Journal of EMDR Practice and Research, 8(2), 74-89. Translated by Miriam Ramos Morrison. 
del reprocesamiento EMDR para tratar los recuerdos traumáticos. En un informe de caso único de una mujer diagnosticada con TLP, Brown y Shapiro (2006) indicaron descensos clínicamente significativos por debajo del punto de corte clínico en todas las subescalas del Inventario de autocapacidades alteradas (Briere, 2004) después de 20 sesiones de terapia EMDR durante un periodo de 6 meses. El funcionamiento de la mujer en la pareja había permanecido inestable después de un período inicial de tratamiento (con el mismo terapeuta) con psicoterapia cognitivo conductual y psicodinámica orientada al conocimiento o insight durante un periodo de 18 meses, que había terminado dos años antes de que finalmente retomase el tratamiento con terapia EMDR.

El tercero de los tres casos, descrito por Wesselmann y Potter (2009), podría cumplir con los criterios para el TLP, a pesar de que la mujer había sido diagnosticada con un trastorno depresivo mayor por su psiquiatra. «La Sra. K describía una depresión aguda y pensamientos suicidas frecuentes, y se autolesionaba cortándose con una cuchilla en los brazos, las piernas o el estómago casi todos los días y, a veces, más de una vez al día» (p. 187). Además, «después de estar en contacto con sus padres, se sentía emocionalmente abrumada y se autolesionaba» (p. 187). Había asistido a tratamiento durante varios años, incluido un tratamiento mientras estuvo hospitalizada por conductas autolesivas, así como 1 año de terapia conductual dialéctica (DBT, siglas en inglés de Dialectical Behavioral Therapy; Linehan, 1993). La Sra. K completó 13 sesiones de terapia EMDR dentro de las 40 sesiones de terapia individual que recibió a lo largo de un año. Durante su terapia EMDR, continuó por separado con las sesiones semanales de DBT. Después de su terapia EMDR, en el seguimiento realizado 1 año después, se observa que había logrado remitir su conducta autolesiva, y sus puntuaciones en la Entrevista de apego adulto (EAA; Hesse, 1999) pasaron de Ds1 (distanciante o despectivo), con una clasificación alternativa de $\mathrm{U} / \mathrm{d}$ (no resuelto/desorganizado), a la F1 (seguro ganado).

\section{Factores etiológicos en el TLP}

\section{Apego y TLP}

Muchos autores han relacionado los trastornos de apego temprano con el desarrollo del TLP, pero diferentes estudios han demostrado que las diversas clasificaciones de apego inseguro son las que están más relacionadas con las características límite en los adultos. La discusión de estos hallazgos se complica por las diferentes medidas utilizadas. Algunos estudios utilizan la EAA con su conjunto dimensional de clasificaciones de apego que incluye una clasificación de no resuelto/desorganizado. Otros estudios utilizan diversos instrumentos de autoinforme con clasificaciones categóricas completamente diferentes que carecen de una clasificación de no resuelto/desorganizado. Agrawal, Gunderson, Holmes y Lyons-Ruth (2004) revisaron estudios de apego de individuos que cumplían los criterios del TLP y concluyeron que los hallazgos más consistentes muestran que estos sujetos tienen estilos de apego no resueltos y temerosos. En los estudios revisados por Agrawal et al. que utiizan la EAA, del $50 \%$ al $80 \%$ de los pacientes con TLP fueron clasificados como «no resuelto». Por otro lado, un estudio reciente con la EAA llevado a cabo por Barone, Fossati y Guiducci (2011) mostró que las categorías de inseguro organizado (distanciante $51 \%$ y preocupado $35 \%$ ) y desorganizado (no resuelto-inclasificable $40 \%$ ) estaban representados en exceso en una visión global de los sujetos con TLP. Además, se encontró una distribución significativamente diferente de la clasificación del apego en cuatro subgrupos de TLP (trastorno concurrente del estado de ánimo/ ansiedad, abuso/dependencia de sustancias, abuso/ dependencia de alcohol y trastornos de la alimentación). En los estudios de autoinforme analizados por Agrawal et al. que incluían una clasificación de "temeroso", el apego preocupado era la segunda categoría con más respaldo entre los sujetos con TLP. Barone et al. encontraron que el apego preocupado era el más frecuente (52\%) en el subgrupo de trastorno del estado de ánimo/ansiedad. En ninguno de los estudios globales de TLP, que incluían la clasificación de no resuelto o temeroso, aparecía preocupado como la clasificación general más prevalente.

\section{Experiencias vitales adversas y TLP}

Diversos estudios han descrito la comorbilidad frecuente entre el TEPT y el TLP (Driessen et al., 2002; McLean y Gallop, 2003; Pagura et al., 2010; Pietrzak, Goldstein, Southwick y Grant, 2011). Otros han encontrado una relación entre el TLP y determinados tipos de abuso (Battle et al., 2004; Cohen, Crawford, Johnson y Kasen, 2005; Golier et al., 2003; Goodman y Yehuda, 2002; Grover et al., 2007; Johnson, Cohen, Brown, Smailes y Bernstein, 1999; Laporte y Guttman, 1996; Sabo, 1997; Tyrka et al., 2009; Yen et al., 2002; Zanarini, 2000; Zanarini et al., 2002). Ball y Links (2009) analizaron la literatura sobre trauma y TLP en el contexto de los criterios clásicos de Hill (1965) para demostrar la causación (fuerza, consistencia, especificidad, temporalidad, gradiente biológico, plausibilidad, coherencia, evidencia experimental y analogía). 
Estos autores demostraron que el trauma se puede considerar un factor causal en el desarrollo del TLP como parte de un modelo etiológico multifactorial.

Un estudio preliminar (Leeds y Mosquera, 2012) con pacientes diagnosticados con TLP exploró retrospectivamente las experiencias tempranas traumáticas y adversas con la Escala de experiencias familiares en la infancia (EFI; Gonzalez, Mosquera y Leeds, 2011). La EFI es un instrumento de autoinforme que abarca diversas experiencias subjetivas y traumáticas durante la infancia dentro de la familia. No es sorprendente que esta investigación encontrarse informes consistentes de experiencias adversas de negligencia emocional y falta de afecto, pérdidas, inversión de roles progenitor-hijo, falta de respeto y conductas agresivas. Experiencias traumáticas más graves, como el abuso sexual, también resultaron ser muy prevalentes (54\% en la muestra de Gonzalez et al., 2011), pero es probable que ambas experiencias de negligencia y de abuso se potencien mutuamente.

Los síntomas límite son similares a las conocidas consecuencias de la traumatización temprana (Timmerman y Emmelkamp, 2001). Varios expertos han propuesto que los síntomas límite se clasifiquen como trastornos de estrés: trastorno de estrés extremo no especificado (DESNOS, por sus siglas en inglés; van der Kolk, Roth, Pelcovitz, Sunday y Spinazzola, 2005), trastorno de estrés postraumático complejo (C-PTSD, por sus siglas en inglés; Herman, 1992) y trastorno de personalidad postraumática (Classen, Pain, Field y Woods, 2006). Numerosas consecuencias características del trauma crónico, severo y temprano se solapan con la sintomatología de la personalidad límite (Driessen et al., 2002; Gunderson y Sabo, 1993; McLean y Gallop, 2003; Roth, Newman, Pelcovitz, van der Kolk y Mandel, 1997; van der Kolk et al., 2005; Yen et al., 2002), lo cual señala una vía causal que va desde las experiencias adversas traumáticas al adulto con TLP. Dado que hay amplia evidencia de las contribuciones del trauma interpersonal grave, crónico y temprano, las perturbaciones del apego y las experiencias vitales negativas en el desarrollo del TLP, parece estar claro el papel del tratamiento EMDR en los individuos con TLP.

\section{Genética y TLP}

Además de la contribución de las experiencias tempranas adversas y traumáticas al riesgo de desarrollar TLP, se han identificado factores genéticos y biológicos en el desarrollo de rasgos límite (Siever, Torgersen, Gunderson, Livesley y Kendler, 2002), así como en el desarrollo de la seguridad del apego y las habilidades de regulación emocional (Brussoni, Jang, Livesley y
Macbeth, 2000; Crawford et al., 2007; Goldsmith y Harman, 1994). En qué medida los factores ambientales y biológicos contribuyen al desarrollo del TLP es una cuestión controvertida (Bakermans-Kranenburg, van Uzendoorn, Bokhorst y Schuengel, 2004; Bokhorst et al., 2003; O’Connor y Croft, 2001). Lo más probable es que el grado de estas influencias varíe en cada caso concreto y que tengan una interacción compleja (Mosquera, González y van der Hart, 2011). Pese a las dificultades actuales para identificar directamente los factores genéticos y biológicos en las personas con TLP, estos aspectos son relevantes para un debate sobre la conceptualización de casos con EMDR y para optimizar el tratamiento farmacológico, que suele ser un abordaje paralelo necesario en personas con TLP. Véase Gianoli, Jane, O’Brien y Ralevski (2012) y Lieb, Völlm, Rücker, Timmer y Stoffers (2010) para análisis recientes de farmacoterapia para personas con TLP. EMDR puede proporcionar una herramienta interesante para ayudar a discriminar el papel de estas primeras experiencias en los problemas actuales de los pacientes, como ha comentado Shapiro (2010).

\section{TLP y disociación}

Las investigaciones han encontrado una alta incidencia de disociación patológica en pacientes con TLP (Chu y Dill, 1991; Galletly, 1997; París y Zweig-Frank, 1997), habiéndose identificado síntomas disociativos en más de dos tercios de las personas diagnosticadas con TLP (Korzekwa, Dell y Pain, 2009; Ross, 2007). Cuando se trabaja con un enfoque EMDR es esencial aclarar, tan pronto como sea posible, si existe disociación estructural significativa (van der Hart, Nijenhuis y Steele, 2006), ya que en estos casos se necesita un «enfoque progresivo» específico para identificar y trabajar las fobias disociativas y acceder de forma segura al material traumático (González y Mosquera, 2012). La relación entre la disociación estructural y el TLP es demasiado compleja como para desarrollarla en este artículo (véase Mosquera et al., 2011, para una revisión completa de este tema).

\section{Consideración de los subgrupos etiológicos del TLP en el plan terapéutico: Aspectos biológicos, de apego y disociativos}

Mosquera y colaboradores (2011) han propuesto tres subgrupos de presentaciones borderline que son relevantes para conceptualizar el caso con EMDR:

1. Un grupo biológico, que tiene factores biológicos más potentes (impulsividad genética o desregulación emocional, trastorno bipolar comórbido o abuso de sustancias). 
2. Un grupo relacionado con el apego, que se basa predominantemente en perturbaciones tempranas de apego.

3. Un grupo disociativo, que cuenta con una historia temprana de apego desorganizado y una historia de traumatización más grave, y presenta un trastorno disociativo comórbido o rasgos disociativos prominentes.

En este artículo abordaremos los dos primeros grupos. Ambos pueden tratarse con procedimientos estándar similares con las adaptaciones descritas en el siguiente texto. La diferencia principal es que el "grupo más biológico» requerirá una intervención más intensiva sobre estos factores y condiciones comórbidas (p. ej., tratar farmacológicamente los rasgos de impulsividad o la desregulación emocional, o proporcionar apoyo específico para el abuso de drogas o alcohol). Al tercer grupo -con rasgos disociativos prominentes- se le trata desde una perspectiva similar a las personas con trastornos disociativos y, como ha señalado Shapiro (2001, p. 443), requiere modificaciones significativas de los procedimientos de EMDR (véase EMDR y disociación: El abordaje progresivo; González y Mosquera, 2012). Estos tres grupos no son excluyentes entre sí, pero pueden ayudar a definir las prioridades del tratamiento y las diferencias en la conceptualización del caso.

Por ejemplo, una paciente con rasgos bipolares comórbidos necesitó un período largo de estabilización farmacológica, seguido de un abordaje progresivo para tratar los recuerdos traumáticos nucleares, alternando el reprocesamiento EMDR con una psicoterapia más de apoyo cuando tenía un estado de ánimo más inestable debido a su vulnerabilidad biológica. Un paciente con síntomas disociativos prominentes y alucinaciones auditivas hostiles necesitó una extensa negociación con las partes disociadas y terapia EMDR centrada en las fobias disociativas antes de comenzar el reprocesamiento EMDR de los recuerdos de experiencias traumáticas. Sin embargo, hay una proporción significativa de pacientes con TLP a los que se puede ofrecer el reprocesamiento EMDR de los recuerdos de experiencias traumáticas al inicio del proceso de tratamiento, y para los que la gravedad de los síntomas o sus respuestas a la instalación del lugar seguro o el DIR no son los principales factores en la decisión de cuándo se debe iniciar el reprocesamiento del trauma.

\section{TLP y EMDR}

El modelo de procesamiento adaptativo de la información (PAI; Shapiro, 2001) proporciona una base conceptual para comprender cómo la información biográfica relevante acerca de las experiencias de la infancia puede estar relacionada con características específicas de la psicopatología adulta, y ofrece un marco terapéutico excelente para un trastorno que, como se mencionó anteriormente, está muy relacionado con las primeras experiencias adversas y traumáticas (Brown y Shapiro, 2006). Sin embargo, se necesitan algunas adaptaciones en los procedimientos estándar de EMDR para trabajar con pacientes con TLP a lo largo de las ocho fases de la terapia EMDR (Leeds y Mosquera, 2012; Mosquera, 2012b; Mosquera y González, 2011a).

\section{Fase 1 en la terapia EMDR:}

Recogida de historia

En el trabajo con el enfoque psicoterapéutico de EMDR, los clínicos necesitan recopilar información acerca de los problemas actuales, así como de las experiencias vitales adversas y traumáticas del pasado. Sin embargo, muchos pacientes con TLP no revelan fácilmente sus problemas actuales o experiencias pasadas más relevantes, a menos que se aborden con cuidado y de forma específica. Es posible que los pacientes no revelen espontáneamente los patrones de apego disfuncionales, el abuso de sustancias, los comportamientos de alto riesgo y otros problemas importantes, ya que pueden estar más centrados en la ansiedad o los síntomas depresivos, o en las dificultades interpersonales actuales. Una herramienta útil para explorar las primeras experiencias relevantes es la escala EFI (González et al., 2011). La escala EFI puede ser útil para explorar cuestiones que los pacientes no reconocen como problemáticas o que se muestran reacios a revelar por vergüenza o por un concepto de defensa de «la privacidad de la familia». La escala EFI va más allá de una simple lista de los 10 recuerdos más traumáticos (Shapiro, 2001, p. 202), y facilita la exploración de una amplia gama de experiencias vitales adversas.

Ajustar el ritmo de la recogida de historia por una capacidad limitada de tolerancia afectiva. Deben tenerse en cuenta los aspectos relacionados con el ritmo y la tolerancia afectiva a la hora de explorar la historia y las dificultades actuales del paciente. Los pacientes con TLP difieren en su capacidad para manejar los sentimientos y los recuerdos que se desencadenan habitualmente al examinar sus estrategias de afrontamiento desadaptativas actuales y sus historias tempranas y adultas, que generalmente son complejas. Por ejemplo, aunque algunos pacientes pueden ser capaces de completar por su cuenta un instrumento de autoinforme como la escala EFI, para muchos esta solitaria tarea resulta abrumadora y desestabilizante. 
Estos pacientes necesitan completar la escala EFI como una entrevista de colaboración a lo largo de varias sesiones, con el apoyo y la orientación del clínico. Los clínicos deben monitorizar activamente el grado en que los pacientes con TLP se sienten activados por el proceso de la recogida de historia y estar listos para frenar y cambiar el foco, para así desarrollar contención, presentificación y habilidades de regulación afectiva. Un abordaje estandarizado de la historia clínica no es adecuado para personas con historias complejas de abandono y de trauma. Lo que puede ser un estilo útil para un paciente, puede ser totalmente inadecuado para otro. Seguir pautas y normas no debería hacernos olvidar la situación y las características específicas de cada paciente. La toma de decisiones en los casos de TLP es más certera cuando se basa en una relación terapéutica bien delimitada y un buen conocimiento del trastorno y del modelo PAI por parte del clínico. Como afirma Shapiro (2001): «Es mejor proporcionar a los profesionales un marco conceptual o modelo que les sirva de guía en su práctica clínica a darles simplemente un procedimiento inflexible paso por paso para aplicar EMDR» (p. xiii). Al trabajar con pacientes con TLP, estas palabras se vuelven especialmente relevantes.

\section{Fase 2: Preparación y estabilización}

La fase de estabilización se ha descrito como esencial antes de pasar al trabajo con trauma (Courtois, Ford y Cloitre, 2009; van der Hart, Brown y van der Kolk, 1989). En el tratamiento del TLP y los trastornos de estrés extremo temprano y crónico, esto supone muchas particularidades que se deben tener en cuenta, entre ellas el papel de los estados mentales relacionados con el apego y las fobias al apego, la emoción y los recuerdos traumáticos (Pearlman y Courtois, 2005; Van der Hart et al., 2006). El trabajo con el TLP y el trauma complejo es intrínsecamente relacional y con frecuencia implica la necesidad de gestionar momentos de emociones intensas y fobias afectivas en la transferencia y la contratransferencia. Es fundamental entender estos aspectos y tener estrategias para abordarlos, tanto antes como durante el reprocesamiento EMDR de recuerdos traumáticos, para asegurarse de que el reprocesamiento de dichos recuerdos pueda hacerse de forma segura y eficaz con estos pacientes (Leeds y Mosquera, 2012).

La relación terapéutica. El aspecto más difícil del tratamiento del paciente con TLP es llegar a establecer una relación terapéutica que a la vez sirva de apoyo y esté bien delimitada con estos pacientes que presentan dificultades pronunciadas en cuanto a apego adulto y conexión interpersonal. Uno de los aspectos más difíciles a la hora de tratar a las personas con TLP es la gestión de las intensas reacciones emocionales que surgen en el terapeuta durante las sesiones de EMDR (Leeds y Mosquera, 2012; Mosquera, 2012b; Mosquera y González, 2011a, 2011b). Los clínicos necesitan tener tolerancia afectiva para continuar reflexionando sobre el significado de las fuertes emociones que pueden sentir ellos mismos cuando los pacientes con TLP expresan emociones vehementes (Dworkin, 2005). Estas respuestas contratransferenciales pueden ser las vías de entrada para llegar a reconocer las cuestiones esenciales que habrán de abordarse con habilidad, o para que se produzcan representaciones involuntarias de las experiencias adversas previas del paciente, del clínico o de ambos (Dalenberg, 2000). Para desarrollar estas habilidades terapéuticas, es importante realizar educación especializada, formación adecuada y supervisión. En algunos casos, los clínicos han de abordar las bases de sus propios problemas de tolerancia afectiva en una terapia personal con EMDR. Como parte del desarrollo de la alianza terapéutica con pacientes con TLP, los clínicos deberían investigar activamente sobre los problemas de los pacientes en sus psicoterapias anteriores para prevenir o minimizar la recurrencia de estas dificultades relacionales del pasado, en la relación terapéutica del presente.

Intervenciones psicoeducativas. Debería proporcionarse psicoeducación al paciente desde la etapa inicial de la terapia (en relación a los mecanismos de defensa, la resolución de conflictos, las emociones, las necesidades y en especial al autocuidado), ayudando al paciente a entender sus problemas, a adquirir perspectiva y a desarrollar los recursos que va a necesitar antes de reprocesar el trauma. Este proceso no necesita ser extenso en todos los casos. De hecho, en algunos casos, la mejor intervención de estabilización puede ser resolver los recuerdos traumáticos relevantes tan pronto como sea posible. Con los pacientes con TLP, la psicoeducación no debe considerarse una intervención limitada en el tiempo, sino algo longitudinal. A menudo es necesario introducir información adaptativa a lo largo de todo el proceso terapéutico. Por ejemplo, durante la historia clínica, en la fase 1 , debe incorporarse información sobre el apego sano, tratarlo más específicamente en el trabajo de autocuidado y volver a presentarlo como entretejido durante las fases $4-7$.

El trabajo con los patrones de autocuidado. Los patrones de autocuidado suelen ser problemáticos en pacientes con TLP. Tienden a carecer de hábitos de autocuidado adecuados y a centrarse en el reconoci- 
miento externo o la satisfacción inmediata. Tienen dificultades para protegerse a sí mismos y para establecer límites sanos. Les falta un equilibrio saludable entre cuidarse a sí mismos y cuidar de los demás. A menudo no pueden tolerar el afecto positivo. Tienen problemas para pedir ayuda: a veces son muy dependientes, otras veces no permiten que los demás les ayuden. Muchas personas con TLP se maltratan internamente, mediante un diálogo negativo consigo mismos o recurren a conductas abiertamente autolesivas. En este grupo clínico, en la fase de preparación es habitual que se tengan que aplicar procedimientos específicos para mejorar los hábitos de autocuidado sanos (González y Mosquera, 2012; Mosquera, 2012a). En casos de trauma complejo, debería ampliarse el DIR (Korn y Leeds, 2002; Leeds, 2009) para incluir procedimientos de autocuidado y desarrollo de habilidades de afrontamiento específicas (González y Mosquera, 2012; Mosquera, 2004), dando prioridad a aquellos objetivos que favorezcan un funcionamiento sano en las actividades normales del día a día: dormir de forma regular, hacer ejercicio de forma regular, comer de manera ordenada, liberarse del abuso de sustancias, y tener apoyo social y estabilidad económica. Se puede hacer una "prueba» para ver cómo responden los pacientes al afecto positivo con el DIR, ya que en contadas ocasiones se han indicado respuestas paradójicas (adversas) a los procedimientos del DIR en pacientes con problemas de tolerancia al afecto positivo (Leeds, 2009, p. 120).

Cómo lidiar con los impulsos deliberados de autolesión y las amenazas de suicidio. De todos los problemas que se pueden encontrar en el tratamiento de los pacientes con TLP, la autolesión deliberada y las amenazas de suicidio son los dos aspectos que a menudo presentan el mayor desafío relacional para el terapeuta. El paciente que se autolesiona o piensa en suicidarse plantea una situación compleja a nivel relacional, y un aspecto central de la respuesta terapéutica es ser conscientes de nuestras respuestas emocionales como terapeutas. La desesperanza y la desesperación de los pacientes que no le encuentran sentido alguno a la vida pueden llevar a que los terapeutas les vean como casos perdidos. Por otro lado, algunos terapeutas pueden implicarse en exceso asumiendo la responsabilidad de la vida del paciente, lo que inevitablemente conduce a un callejón sin salida en el proceso terapéutico. La autolesión deliberada y las amenazas de suicidio son problemas multifacéticos que pueden estar relacionados con la falta de regulación emocional, con patrones negativos de autocuidado, con culpa no procesada y con cuestiones relacionales.
El manejo de estas cuestiones ha de desarrollarse como parte de una conceptualización integral del caso, ya que pacientes diferentes con comportamientos similares pueden responder de manera favorable o no a las mismas intervenciones. Un paciente que amenaza con suicidarse o lo intenta, porque se siente incapaz de gestionar sus problemas actuales, puede que responda mejor a una intervención familiar para movilizar más apoyo. En otros casos de amenazas de suicidio, la intervención correcta sería desarrollar mejores procedimientos de autocuidado y límites más robustos para reducir su dependencia de los demás y ayudarle a centrarse en sus propios recursos. Un paciente que tiende a hacerse cortes porque carece de capacidades reguladoras adecuadas podría beneficiarse de un abordaje psicoeducativo, como describe el apartado siguiente sobre regulación emocional. En otros casos en los que la conducta autolesiva está muy relacionada con pensamientos irracionales de culpa (p. ej., «es mi culpa»), la intervención de estabilización más potente puede ser reprocesar un recuerdo nuclear asociado a esta cognición negativa. Las dianas para el reprocesamiento en estos casos no son las conductas autolesivas en sí, sino más bien las circunstancias que rodean el primer momento en que se produjo una autolesión, el origen de las creencias negativas asociadas al problema, y los recuerdos que pueden ser identificados a través de un puente afectivo, cognitivo o de impulso de acción (Leeds, 2009; Watkins, 1971, 1990).

$\mathrm{Al}$ igual que en cada uno de los procedimientos de EMDR realizados con pacientes con TLP, una relación firme, de apoyo y bien delimitada es crucial para el manejo de estas situaciones complicadas. Al paciente se le ha de considerar responsable de sus decisiones y comportamientos, aunque ha de seguir sintiendo que tiene el apoyo del terapeuta. Debe desarrollarse una estrategia de tratamiento de las autolesiones y amenazas de suicidio para cada caso concreto, junto con el paciente y cualquier familiar involucrado.

\section{Desarrollo de capacidades de regulación emocio-} nal. Los pacientes con TLP son propensos a estados emocionales intensos que se sienten incapaces de manejar, o que se amplifican a través de reacciones emocionales secundarias (p. ej., enfadarse porque se sienten tristes). Por un lado, parecen tolerar un amplio rango de reactividad emocional y, sin embargo, al mismo tiempo suelen demostrar una tolerancia extremadamente limitada para determinadas emociones. Los pacientes con TLP necesitan aprender a aceptar todas sus reacciones emocionales y colocarlas en los contextos situacionales para que puedan entender lo que las desencadena (incluidos acontecimientos pa- 
sados y presentes). La psicoeducación acerca de las emociones y sus funciones es crucial para que se den cuenta de que sentirse dolidos y enfadados no quiere decir que sean "repugnantes», "defectuosos» o que estén «equivocados».

En las primeras etapas de la terapia, es esencial fomentar la autoobservación, ya que los pacientes con TLP tienden a sentirse abrumados por sus emociones y no reflexionan sobre su importancia. El desarrollo de una perspectiva metacognitiva (Fonagy, Gergely, Jurist y Target, 2002) es un logro regulatorio básico en los pacientes con TLP, quienes de esa manera pueden llegar a ser conscientes de sus reacciones. Esta «metaperspectiva» también es fundamental para facilitar la capacidad de atención dual (al pasado y al presente) necesaria para un reprocesamiento EMDR eficaz del recuerdo en las fases de la 4 a la 7 . Por ejemplo, una paciente con TLP se enfadó con ella misma después de abrirse emocionalmente a alguien que, posteriormente, la traicionó. Refirió que su emoción inicial fue de tristeza, pero que inmediatamente comenzó a insultarse a sí misma por ser débil y emocional. Se le pidió que notase qué cambiaba, en caso de hacerlo, si contemplaba su tristeza sin juicio y con total aceptación, y luego se hizo una tanda de EBL. En ese momento comentó que era capaz de sentir compasión hacia sí misma. Con otra tanda de EBL, su compasión hacia sí misma aumentó y comenzó a pensar en las señales de advertencia de que esa relación no era de fiar. Luego añadió que podría aprender de esta experiencia.

Cuando los pacientes señalan un miedo excesivo a las reacciones adversas de los demás ante unos límites apropiados, o conductas dirigidas hacia sí mismos, los orígenes de estos temores se pueden identificar a través de la historia clínica o el uso de un puente afectivo y, a continuación, reprocesarse. Las actividades de autodistracción pueden ser más útiles que el trabajo de contención, debido a que estos pacientes habitualmente tienden a contenerse demasiado hasta que explotan. Las estrategias de regulación impulsadas desde el exterior (llamar desesperadamente a amigos o familiares, buscar el amor romántico «verdadero», etc.) deberían cambiar gradualmente para aprender a autorregularse (a través del diálogo interno de apoyo, cambiar el foco de atención, etc.). Las soluciones mágicas deberían transformarse en estrategias realistas. Los procedimientos de autocuidado pueden ayudar a estos pacientes a entender sus estados emocionales, a aceptarlos y verse a sí mismos con compasión y sin juicios, y a establecer límites en las relaciones. Las intervenciones de regulación emocional no deberían entenderse meramente como una fase de preparación del tratamiento previa al comienzo del reprocesamiento. Ambas intervenciones se pueden combinar de forma dinámica en función de las características específicas de cada paciente. En algunos casos, la mejor intervención de autorregulación es reprocesar experiencias traumáticas nucleares sin resolver, relacionadas con ser invisible, no ser atendidos o no tener a nadie a quien pedir ayuda y protección.

Trabajo focalizado en el abuso de sustancias. El abuso de alcohol y drogas son, con frecuencia, trastornos concurrentes en el TLP (Paris y Zweig-Frank, 2001; Trull, Sher, MinksBrown, Durbin y Burr, 2000; Zanarini, Frankenburg, Hennen, Reich y Silk, 2004; Zimmerman y Mattia, 1999) que deben explorarse específicamente. El abuso de sustancias suele estar relacionado con las limitadas capacidades de autorregulación emocional observadas en individuos con TLP, y es un ingrediente habitual en la miríada de complejas interacciones que confunde el tratamiento de la patología límite, y por la que se requieren abordajes específicos. Para favorecer el desarrollo de la abstinencia puede estar indicada una derivación a un grupo de tratamiento basado en la evidencia para el abuso de sustancias, tal como la terapia de búsqueda de la seguridad (Najavits, 2002; Najavits, Weiss, Shaw y Muenz, 1998), durante las primeras fases del tratamiento, paralelamente al desarrollo de la relación terapéutica y el plan de tratamiento. También puede que sea necesario integrar en el plan de tratamiento una terapia individual EMDR centrada en el desarrollo de habilidades de afrontamiento (Korn y Leeds, 2002) y en el tratamiento de los disparadores actuales de las recaídas (Hase, 2010; Hase, Schallmayer y Sack, 2008; Hofmann, 2004, 2010; Popky, 2005).

Algunos pacientes pueden necesitar derivaciones a programas de tratamiento residenciales o ambulatorios al comienzo de su tratamiento individual, y así recibir apoyo para llegar a la abstinencia o superar el riesgo de comportamientos peligrosos relacionados con el abuso de sustancias (p. ej., coma etílico o sobredosis de drogas). En otros casos, puede que las experiencias previas en estos programas no hayan sido eficaces y necesiten terapia EMDR centrada en los disparadores de las recaídas en el presente (Hase, 2010; Hase et al., 2008; Hofmann, 2004, 2010; Popky, 2005) o incluso en los recuerdos traumáticos tempranos pertinentes. Por ejemplo, un paciente de 43 años de edad bebía compulsivamente y presentaba serias conductas agresivas cuando estaba bajo los efectos del alcohol. El riesgo de hacerle daño a alguien era alto, por lo que su terapeuta lo derivó a un programa residencial de tratamiento de la alcoholemia. Después de un mes en el hospital, fue expulsado 
por frecuentes problemas interpersonales con otros pacientes. Su terapeuta decidió intentar hacer reprocesamiento EMDR sobre un recuerdo temprano conectado con una sensación de impotencia y culpa que precedió a sus peores episodios de alcoholismo. El éxito del reprocesamiento de ese recuerdo puso fin a los comportamientos problemáticos e hizo que le fuera posible continuar su tratamiento ambulatorio. En casos como este ejemplo, un trabajo conservador con las tareas de la fase de preparación para el desarrollo de habilidades y de autorregulación puede ser insuficiente a la hora de satisfacer la necesidad de un paciente de abordar uno o más recuerdos tempranos que subyacen a las conductas reactivas o a las recaídas recurrentes.

El trabajo con las defensas. En el sentido más amplio, las defensas psicológicas implican una gama de conductas de afrontamiento internas y externas que sirven para evitar sentimientos e impulsos conflictivos (McCullough, 2003). Las defensas pueden ser entendidas como protecciones, como fobias afectivas (McCullough, 2012) o fobias interoceptivas de acciones mentales derivadas del trauma (van der Hart et al., 2006). No se debe confrontarlas ni evitarlas de manera forzada. Más bien, el terapeuta debería acercarse a ellas progresivamente a un ritmo que el paciente puede tolerar (González y Mosquera, 2012; McCullough, 1997). La psicoeducación es una intervención fundamental en este sentido. McCullough (2012) describe este paso como el reconocimiento de la defensa.

Las defensas pueden convertirse a menudo en aliados de la terapia cuando paciente y terapeuta comprenden su función subyacente. Cuando el paciente es capaz de identificar una defensa que el terapeuta cree que está bloqueando el proceso terapéutico, es posible a veces reprocesar la defensa con intervenciones específicas de EMDR (González y Mosquera, 2012 ; Knipe, 1999, 2003, 2005, 2009, 2010a, 2010b, 2010c; Leeds, 2009, p. 164; Leeds, 2012; Shapiro, 2001, pp. 192-197). Por ejemplo, un paciente presentaba frecuentes actitudes defensivas en terapia, tales como minimización, tendencia a entablar conversaciones superficiales y evitación, y cancelar o llegar tarde a sus citas. El terapeuta identificó sus tendencias de evitación como un tema central y las seleccionó como disparadores actuales para que pudieran ser reprocesadas. Después de esto, mejoró su cumplimiento terapéutico y su participación.

Procedimientos específicos para la autodiferenciación. Un aspecto relevante que debe abordarse es la autodiferenciación. Harter (1999) analiza diferentes perspectivas acerca de la construcción del autocon- cepto y propone una perspectiva evolutiva que explica cómo la interacción temprana con los cuidadores (con frecuencia alterada en los pacientes con TLP) da forma a las representaciones adultas de uno mismo. Muchas personas con TLP presentan una falta de diferenciación del self, en la que la percepción de uno mismo contiene una mezcla de emociones propias y de otras personas. Es decir, en el self indiferenciado de los pacientes con TLP, hay una coincidencia entre las percepciones internas y externas de las emociones. Kernberg (1993) describe intervenciones para abordar esta cuestión como primer paso para el tratamiento de las personas límite. Bateman y Fonagy (2004) llaman a este tema central la «equivalencia psíquica». Se han propuesto diversos procedimientos específicos de EMDR para la diferenciación (Litt, 2007).

El trabajo con las partes disociativas de la personalidad. Por lo general, encontramos grados de disociación estructural (van der Hart et al., 2006), incluso en pacientes con TLP con síntomas disociativos más leves. Las partes disociadas de la personalidad pueden ser percibidas y descritas por los pacientes como «diferentes aspectos» o como "reacciones cambiantes». La exploración de la presencia de las partes disociadas de la personalidad a través de representaciones concretas (dibujos, pequeñas figuras, etc.) es útil para que los terapeutas puedan desarrollar y refinar una formulación del caso en más profundidad y para que los pacientes desarrollen habilidades de pensamiento reflexivo y una perspectiva metacognitiva (van der Hart, Groenendijk, González, Mosquera y Solomon, 2013, pp. 8-9). Un entendimiento compartido de estos diversos aspectos de la personalidad es esencial para el éxito de reprocesamiento del trauma con EMDR (González y Mosquera, 2012). En los casos en que la disociación estructural no es prominente, no es necesario trabajar con partes disociadas de la personalidad durante mucho tiempo, pero una exploración específica de estos aspectos en la fase de preparación a menudo ayuda a prevenir o a resolver dificultades durante las fases 3 a 7.

\section{Reprocesamiento del trauma: Fases 3-7}

Con la mayoría de las personas con TLP que presentan en la actualidad habilidades de afrontamiento limitadas y una estabilidad emocional mínima, generalmente es mejor no comenzar el reprocesamiento por sus peores recuerdos o por ningún recuerdo temprano traumático o adverso. En muchos de estos casos que presentan una menor estabilidad emocional, sin embargo, podemos empezar a utilizar procedimientos estándar de EMDR en fases relativamente 
tempranas de la terapia con los disparadores actuales (Hofmann, 2010; Leeds y Mosquera, 2012; Mosquera, 2012b), tales como la reactividad emocional frente a una pareja actual, un compañero de trabajo o un supervisor. Con pacientes con TLP que presentan una mayor estabilidad emocional, en ocasiones es posible comenzar a reprocesar los recuerdos antiguos, pero esto no es lo habitual.

Ajustar el ritmo del proceso terapéutico. Al iniciar el reprocesamiento, el proceso de toma de decisiones para la selección y secuencia de dianas puede estar influenciado por muchos factores, tanto por parte del terapeuta como del paciente (Leeds y Mosquera, 2012). Algunos clínicos (bajo la influencia de una contratransferencia inicial idealizada de rescatar o salvar al paciente) pueden tender a pasar demasiado rápido a descubrir material traumático sin entender primero la capacidad del paciente para hacer frente a las dificultades del día a día, así como sus dificultades para enfrentarse al material traumático. Por otro lado, el paciente o el terapeuta pueden evitar o retrasar de forma defensiva el reprocesamiento del trauma debido a una fobia afectiva o a una fobia a los contenidos traumáticos (McCullough, 1997; van der Hart et al., 2006).

\section{Cómo determinar (probar) la preparación para el procesamiento}

Probar los pasos del procedimiento estándar de $E M D R$. Se puede iniciar un intento prudente de utilizar el protocolo estándar EMDR completo para el TEPT (Leeds, 2009; Shapiro, 2001) cuando el paciente muestra signos de estabilidad (como se describe en el siguiente apartado). Este intento inicial puede ser etiquetado como prueba, para ver si es un buen momento para empezar a abordar directamente los recuerdos negativos y traumáticos tempranos. Si el primer objetivo está bien elegido, si el paciente entiende el objetivo del procedimiento y tiene unas expectativas realistas, los resultados de esta prueba pueden guiar las decisiones para avanzar hacia el reprocesamiento de otras dianas.

Probar los pasos del procedimiento de EMDR modifcado. Se puede ofrecer una prueba preliminar, incluso a los pacientes con un funcionamiento bajo, modificando ligeramente los pasos del procedimiento estándar de EMDR. Esta prueba puede consistir en un reprocesamiento corto e incompleto de un elemento negativo: una situación incómoda en la vida diaria o un recuerdo del pasado reciente con poca perturbación (bajo nivel de SUD). Aquí, es importante volver a la diana después de unas tandas de EBL (limitando así las asociaciones) y detener el procedimiento tan pronto como la perturbación disminuya, incluso aun- que sea un poco, o aparezcan algunas revelaciones o insights. Este breve ensayo debería durar solo unos minutos. Se le puede presentar al paciente como un experimento para probar los efectos de los procedimientos de EBL sobre las experiencias perturbadoras negativas y decidir conjuntamente si los procedimientos de EBL pueden ser útiles al inicio del tratamiento o si se deben retrasar.

El terapeuta debe explicar al paciente que, incluso sabiendo que la terapia EMDR con EBL puede ser útil en su caso, es muy importante encontrar el momento exacto para hacerlo. El terapeuta debe describir el "experimento" como una situación con muchos resultados posibles: las sensaciones positivas y negativas pueden aumentar, disminuir o quedarse igual, y pueden cambiar de lugar (localización sentida en el cuerpo) o no. La EBL puede desbloquear situaciones que están atascadas en el cerebro del paciente, y esto puede notarse de diferentes maneras: el paciente puede llegar a ser más consciente de sus emociones, las puede notar como más o menos intensas, pueden venirle diferentes recuerdos... y todo esto está bien. Todas estas posibles respuestas aportarán información sobre cómo procesa la información el cerebro del paciente.

Con los resultados de esta prueba, el paciente y el terapeuta sabrán si los procedimientos de reprocesamiento EMDR pueden ser introducidos al principio del tratamiento o si se necesita una fase de preparación más larga antes de volver a intentar la prueba unos meses más tarde. El terapeuta debe insistir en que el paciente no fuerce nada; esto va a mostrar un gran respeto por la opinión del paciente en la toma de decisiones. Incluso cuando el experimento muestre que el reprocesamiento EMDR puede ser angustioso para este paciente en particular, la relación terapéutica puede fortalecerse porque la persona aprende que la terapia se basa en una postura de colaboración, donde el paciente es el protagonista principal.

La selección de dianas. En el trabajo con pacientes con TLP, la selección de dianas puede ser un reto por varias razones. Puede haber dianas que procedan de muchas experiencias de vida adversas y muchas experiencias traumáticas únicas o recurrentes (Leeds y Mosquera, 2012; Mosquera, 2012b). Por otro lado, en los casos de trauma complejo, la amnesia o los recuerdos fragmentados pueden interferir en la identificación de dianas cruciales del pasado. Cuando hay recuerdos identificados claros, es posible que los mecanismos de defensa y de evitación no permitan que se trabaje con ellos directamente. En su lugar, puede ser necesario hacer más preparación, psicoeducación 
y trabajo de estabilización. No es infrecuente que la evitación defensiva y fóbica que rodea a ciertos recuerdos (van der Hart et al., 2013) tenga que ser la diana inicial para el reprocesamiento, hasta que se resuelvan suficientes miedos concretos y se desarrolle suficiente tolerancia afectiva.

Cómo determinar la preparación. De acuerdo con el modelo consensuado orientado por fases (Courtois et al., 2009; van der Hart et al., 1989), Hofmann (2010) propuso que, antes de proceder a destapar y reprocesar recuerdos traumáticos nucleares, por lo general, los pacientes con TEPT complejo con una inestabilidad significativa deberían cumplir los siguientes cuatro criterios de estabilidad: un buen autocuidado en el funcionamiento del día a día, recursos de contención y autocalmantes, conexión con las sensaciones corporales y la capacidad de poder hablar de un recuerdo traumático sin desregularse. Hofmann propuso que a los pacientes con una inestabilidad signficativa con TEPT complejo que no cumplan con estos criterios se les ofrezca DIR (Korn y Leeds, 2002), Instalación constante de orientación y seguridad en el presente (CIPOS; Knipe, 2010d) y reprocesamiento de las dianas de la vida cotidiana (disparadores actuales) hasta que consigan una mayor estabilidad. Los terapeutas que eligen constantemente esperar a que se den estas cuatro condiciones antes de comenzar con el reprocesamiento del trauma con EMDR pueden terminar esperando años, o tal vez nunca lleguen a la «estabilidad necesaria» en absoluto. Este modo "conservador» de utilizar el método EMDR puede retrasar o impedir indebidamente que muchos pacientes con TLP se beneficien del núcleo de la terapia EMDR (González y Mosquera, 2012).

En algunos casos, cuando los pacientes con TLP carecen de buenas habilidades de autocuidado y autorregulación, el reprocesamiento del trauma puede llegar a tolerarse bien y ser eficaz. Estos pacientes pueden llegar a regularse mejor después de un reprocesamiento limitado del trauma, mientras que otras intervenciones «de menor intensidad», que aplazan o evitan la confrontación con el recuerdo del trauma como el DIR o la DBT- puede que no funcionen tan bien con ellos. En algunos casos, no hay otros recursos disponibles para la persona o se está quedando sin opciones. Aquí, la intervención más estabilizadora puede consistir en identificar las experiencias adversas y traumáticas de la vida claramente vinculadas a las conductas de mayor riesgo y a los problemas más debilitantes, y reprocesarlas pronto en la terapia. Con algunos pacientes, lo mejor es "ir despacio» y seguir abordajes prudentes. En otros casos, se necesita un enfoque más directo. La información de las "pruebas terapéuticas» es necesaria para diseñar una formulación integral del caso.

Debido a que los pacientes que cumplen con los criterios de TLP pueden ser muy diversos en cuanto a su organización individual y su capacidad de respuesta a nuestras intervenciones, hay que proponer cada intervención como un experimento y considerar cuidadosamente sus respuestas en y entre sesiones, como información esencial para la toma de decisiones clínicas. A medida que comprobamos que los pacientes con TLP toleran nuestras intervenciones y logran mayor estabilidad, pasamos a reprocesar las dianas más relevantes y, en ocasiones, dianas cada vez más perturbadoras. A continuación se describirán algunas propuestas para estructurar las dianas y el reprocesamiento.

Distintas vías en el plan de tratamiento para el TLP. Los pacientes con TLP presentan diferentes situaciones específicas que influyen en el camino que seguirá el proceso terapéutico. Con el abordaje progresivo, podemos empezar a trabajar partiendo de tareas más tolerables hacia otras más difíciles que utilizan la terapia EMDR con EBL. En general, cuando se trabaja con los pacientes con TLP (aparte del subtipo disociativo), se pueden considerar cinco opciones básicas de intervenciones EMDR desde la más tolerable y accesible hasta la de más difícil acceso y tratamiento:

1. Ofrecer psicoeducación y DIR. Esto es apropiado cuando el terapeuta valora con precisión que el paciente necesita una preparación específica y no está listo para el reprocesamiento de elementos disfuncionales.

2. Explorar en colaboración los efectos de la EBL sobre elementos disfuncionales limitados, solo para probar cómo funciona la EBL en este paciente en concreto. Estos elementos incluyen sensaciones desagradables y situaciones de la vida diaria difíciles, pero no demasiado complicadas.

3. Hacer diana sobre elementos defensivos disfuncionales limitados y selectivos, y reprocesarlos. Esto se diferencia de un reprocesamiento completo de la red de memoria de recuerdos específicos. Algunos ejemplos incluyen hacer diana sobre impulsos defensivos de evitación, impulsos compulsivos de distracción o material de transferencia.

4. Hacer diana sobre los disparadores actuales y reprocesarlos (mientras se podan o posponen deliberadamente los recuerdos nucleares). Estos se seleccionan cuando el terapeuta valora que el paciente aún no está listo para trabajar recuerdos antiguos, pero está listo para reprocesar. 
5. Hacer diana sobre los recuerdos antiguos y reprocesarlos. Estos se seleccionan cuando el terapeuta valora que el paciente está listo para trabajar recuerdos antiguos. Estos pueden ser recuerdos del inicio de la edad adulta, la adolescencia o la infancia, en función de su contribución al peor de sus síntomas actuales. Podemos encontrarlos a través de (a) la historia clínica directamente, (b) el puente afectivo (Watkins, 1971, 1990), el «float-back» (Young, Zangwill y Behary, 2002, p. 195) o los disparadores actuales y (c) la asociación espontánea desde los recuerdos más recientes a un recuerdo nuclear anterior.

Caracteristicas del paciente que guian la secuencia de dianas. Se deben tener en cuenta determinadas características de los pacientes para orientar la secuencia de dianas y para organizar un plan terapéutico. Estos son algunos ejemplos del proceso de toma de decisiones:

Si hay conductas de alto riesgo o sintomas que conducen a que el paciente se desestabilice emocionalmente. Para favorecer que el paciente logre la estabilidad, comenzamos con dianas del presente o del pasado que están claramente asociadas con los síntomas más debilitantes del paciente, y continuamos trabajando con aquellas asociadas a esos síntomas específicos hasta que el paciente muestre beneficios estables, con una reducción o eliminación de los síntomas asociados. Se deben abordar en primer lugar las autolesiones, las conductas agresivas o las conductas de alto riesgo, o las dianas relacionadas con la descompensación emocional del paciente. Cuando el paciente pueda tolerar hablar de los recuerdos tempranos, incluso de los que tienen emociones intensas, comenzamos a trabajar en un recuerdo temprano que tanto paciente como terapeuta entiendan que está conectado con las conductas de alto riesgo o los problemas debilitantes. Cuando los pacientes demuestran que aún no pueden tolerar el focalizarse en dianas más perturbadoras del pasado, nos centramos en el reprocesamiento de los disparadores actuales y se alterna con un breve contacto espontáneo o guiado con dianas del pasado, hasta que el paciente puede tolerar un contacto más amplio con el pasado.

Si hay creencias, pensamientos o recuerdos intrusivos. Cuando hay recuerdos intrusivos, trabajamos primero sobre ellos. Cuando las intrusiones de los recuerdos son intensas y recurrentes, reprocesarlas puede beneficiar mucho al paciente. A menudo, este tipo de recuerdos intrusivos están tan activados que intentar reprocesar cualquier otro material sería ineficaz. Los pensamientos negativos persistentes o las creencias limitantes sobre uno mismo pueden ser una diana inicial productiva, seguidos de la búsqueda de un recuerdo específico mediante los procedimientos descritos anteriormente.

Cuando ya no quedan conductas de alto riesgo o creencias, pensamientos o recuerdos intrusivos. Cuando las conductas de alto riesgo y las intrusiones se han resuelto o están ausentes, en general, las dianas se deben seleccionar en base a la capacidad del paciente para tolerar el trabajo con recuerdos tempranos y perturbadores.

Cuando los pacientes indican haber conectado con recuerdos activados (Korn, 2009), lo mejor es seleccionar un recuerdo en el que al paciente le gustaría trabajar para disminuir la perturbación asociada. Esta es siempre mejor opción que un recuerdo que el terapeuta cree que es relevante en aquellas situaciones en las que (a) no hay un acuerdo paciente-terapeuta acerca de la relación del recuerdo con los problemas actuales del paciente, y cuando (b) el paciente no está dispuesto a trabajar en el recuerdo que prefiere el clínico ni está preparado para ello.

Cuando los pacientes no conectan con los recuerdos perturbadores tempranos o hay una amnesia disociativa, se comienza el reprocesamiento de los disparadores actuales asociados.

En el trabajo con pacientes con TLP, donde es común «diseminar la activación» a muchas experiencias adversas asociadas (Collins y Loftus, 1975; Leeds, 1998) y la capacidad de tolerancia afectiva se mantiene reducida, suele ser importante limitar el alcance del material asociado que aparece durante el reprocesamiento de un disparador actual. Cuando surge material asociado, generalmente el clínico debería responder reconociendo el significado de las dianas anteriores y volviendo a prestar atención al disparador actual. Sin embargo, si el clínico cree que ha surgido un recuerdo tolerable, puede considerar hacer una prueba y continuar el reprocesamiento con ese recuerdo, solo después de pedirle permiso el paciente para continuar y solo mientras el reprocesamiento centrado en el recuerdo siga siendo tolerable.

Cuando surgen recuerdos que son intolerables: (a) dejarlos a un lado; (b) si es necesario, ayudar al paciente a volverse a estabilizar; (c) continuar con el disparador actual; y (d) solo si el paciente está dispuesto, reanudar el reprocesamiento. El clínico debería estar alerta a las señales indirectas de malestar (como inquietud creciente, agitación o capacidad reducida para seguir suavemente los movimientos oculares). Dado que los pacientes con una disociación estructural más grave pueden no ser conscientes de este creciente malestar o no mostrar señales observables, el clínico debería estar 
preparado para buscar señales de stop implícitas de otras partes del sistema de la personalidad, por ejemplo mediante el uso del escáner interno (González y Mosquera, 2012). Es preferible usar unidades de trabajo más pequeñas. Lo más importante es que el paciente permanezca dentro de la ventana de tolerancia y que nos adaptemos a la energía mental que posee en cada momento. En casos de trauma complejo con historias tempanas de indefensión aprendida, es esencial y muy terapéutico dar a los pacientes un papel activo en la toma de decisiones, siempre sin forzar y comprobando periódicamente para asegurarse de que no están abrumados o agotados.

Cuando el paciente está listo para reprocesar recuerdos traumáticos tempranos. Cuando el paciente está (a) estable y lo suficientemente fuerte como para hacer frente a los recuerdos traumáticos tempranos y muy perturbadores, (b) no hay defensas importantes o amnesia disociativa y (c) se ha realizado una primera prueba experimental con EBL, podemos hacer diana directamente sobre esos recuerdos, pero generalmente se recomiendan primero los abordajes progresivos descritos anteriormente.

Adaptaciones en la fase de evaluación. Después de haber seleccionado una diana propuesta para el reprocesamiento, es importante permanecer alerta a las precauciones adicionales durante la fase 3 . En el trabajo con la selección de las cogniciones negativas y positivas a veces es necesario que el terapeuta ayude y lo simplifique. Aunque es útil saber qué cognición negativa está relacionada de manera más intensa con la diana, es fundamental evitar que los pacientes con TLP se pierdan totalmente en una cascada creciente de autocreencias negativas. Los pacientes con TLP suelen tener muchas auto creencias negativas fuertes. A menudo tienen dificultades cuando se trata de seleccionar solo la que está más conectada con una experiencia particular (Leeds y Mosquera, 2012; Mosquera, 2012b). Para desarrollar la capacidad de mentalización (Bateman y Fonagy, 2004) en los pacientes con TLP, es muy terapéutico que entiendan que su autoconcepto ha surgido a partir de experiencias concretas (externas). Por lo que este aspecto estándar de la Fase 3 es una intervención altamente relevante y potencialmente adaptativa que debe mantenerse en el procedimiento EMDR estándar. Sin embargo, los pacientes que tienden a sentirse desbordados con este paso necesitan más ayuda del terapeuta para evitar sentirse abrumados o perdidos. Por ejemplo, el terapeuta podría decir: «Sé que esto es difícil y que te pueden estar viniendo muchas afirmaciones negativas ahora, pero por favor, trata de pensar en la situación y escoge la afirmación que encaje mejor. Tómate el tiempo que necesites». O el terapeuta podría decir: "Cuando te enfocas en ese recuerdo y en los sentimientos que te vienen, ¿están más relacionados con una sensación de falta de control, una sensación de peligro inmediato, o una sensación de no valía?» Al mismo tiempo, los terapeutas tienen que estar alerta para evitar hacer el trabajo por el paciente en este paso, como ofrecerles inicialmente una cognición negativa específica. Puede incluirse información psicoeducativa, y debería estimularse el pensamiento reflexivo.

En los pacientes con TLP no pocas veces puede ser más difícil identificar una cognición positiva $(\mathrm{CP})$ que identificar una negativa. Es posible que los pacientes que carecen de experiencias tempranas de afecto positivo interpersonal compartido y reconocimiento (Leeds, 2006) carezcan de tolerancia al afecto positivo o autoesquemas positivos, incluso con las autoafirmaciones más sencillas. En algunos casos, el trabajo específico relativo a la tolerancia al afecto positivo puede ser útil antes de proceder a reprocesar el trauma. En otros casos, la CP propuesta inicialmente por el paciente puede ser una creencia idealizada y poco realista, que debe ser modificada con orientación del terapeuta mediante una conversación de colaboración con el paciente. Ejemplos sencillos serían: «nunca ocurrió", "puedo tener todo bajo control» o «soy invencible». Un ejemplo más sutil podría ser un paciente que dice como CP «soy fuerte», cuando la cognición negativa ha sido «no es seguro para mí mostrar mis necesidades o pedir ayuda». Aquí, la idea de ser "fuerte» es en realidad una defensa contra la vulnerabilidad de pedir lo que uno necesita. En cualquier caso, se debe seleccionar una CP adecuada antes de pasar a la fase 4 (desensibilización), ya que si no el paciente estará expuesto a un reprocesamiento que va de negativo a negativo, sin conectar en ningún momento con redes de memoria positivas. Si no es posible encontrar una CP fuerte y específica («soy buena persona»), se puede escoger una que sirva de puente, por ejemplo, «puedo aprender a aceptarme como soy».

\section{Adaptaciones en la fase de desensibilización}

Crear una estructura frente a la propagación de la activación. Un aspecto fundamental durante la desensibilización en la fase 4 para los pacientes con TLP es la necesidad de mantener una estructura (foco). Estos pacientes son propensos a que se «disperse la activación» (Collins y Loftus, 1975; Leeds, 1998), ya que una red de memoria disfuncional se enlaza con muchas otras y rara vez conectan con redes de memoria adaptativas. Por lo tanto, la pauta estándar de EMDR al inicio del reprocesamiento de decir «deja que venga 
lo que tenga que venir» puede no ser efectiva en los casos de trastorno de estrés extremo. Estos pacientes tienden a vivir en el caos y pueden tener una lista de los «top 100» recuerdos perturbadores en lugar de una lista de «top 10». En muchos pacientes con TLP, si el clínico se limita a seguir el proceso asociativo espontáneo, fácilmente se puede activar demasiado material sin llegar a un reprocesamiento eficaz. Diversos autores (Gelinas, 2003; Korn, 2009; Leeds, 2009; Paulsen, 1995) han propuesto variaciones en las pautas de reprocesamiento estándar que, de alguna manera, se parecen a las del EMDR original (Shapiro, 1989) -que volvía a la diana después de cada tanda de EBL- utilizando cadenas asociativas cortas. Después de solo dos o tres tandas de EBL, o tan pronto como surgen por asociación espontánea uno o dos recuerdos nuevos (negativos), se vuelve a la diana y se pregunta «QQué es diferente?» (para evitar la respuesta automática de «lo mismo»).

El uso repetido de entretejidos para activar información adaptativa. La activación deliberada de información adaptativa, también conocida como entretejido (Leeds, 2009) o entretejido cognitivo (Shapiro, 2001), se puede introducir repetidamente antes de realizar más tandas de EBL cuando sea necesario abordar un reprocesamiento ineficaz. El terapeuta puede introducir algunos elementos de un cambio positivo en las creencias, por ejemplo, «QQué te ayudaría a pensar mejor sobre ti mismo?» Para ayudar al paciente a avanzar hacia el pensamiento reflexivo, la mentalización y la realización o «darse cuenta», cuando se vuelve a la diana el terapeuta puede hacer una de las siguientes preguntas: «QQué es lo que entiendes acerca de esta experiencia ahora?», «QQué piensas acerca de esta experiencia ahora?», «¿De qué eres consciente ahora que no eras consciente entonces?» Durante el reprocesamiento con pacientes con TEPT por un número único o limitado de experiencias, la vinculación de información adaptativa y el darse cuenta de ello suelen ocurrir espontáneamente. Durante el reprocesamiento con pacientes con trastorno de estrés extremo, es posible promover activamente la vinculación de la información adaptativa y la realización que, por la limitada exposición temprana a experiencias adaptativas, puede que no tenga lugar de otro modo.

Exploración activa de las emociones secundarias que pueden alterar el reprocesamiento. Otra forma de mejorar el reprocesamiento efectivo es ayudar al paciente a lidiar con las emociones secundarias que surgen con frecuencia en los pacientes con TLP. Estas emociones secundarias están relacionadas con juicios negativos o creencias que se activan como consecuencia de un estado emocional primario (véase Leeds, 2009, p. 164;
Shapiro, 2001, pp. 192-193). Por ejemplo, cuando el paciente experimenta rabia, puede pensar «soy malo (igual que lo era el abusador enfadado)». La creencia "soy malo" se asocia con emociones secundarias de vergüenza. Esta emoción secundaria puede alterar el reprocesamiento. Dichas disrupciones pueden hacerse visibles mediante declaraciones tales como «no me llega nada», síntomas somáticos repentinos, hablar en exceso, aumento de la ansiedad o aumentos abrumadores del nivel de activación emocional del paciente.

Después de cada tanda de EBL, después de hacer la pregunta estándar (“¿Qué estás notando ahora?») y escuchar la respuesta del paciente, cada vez que el terapeuta observa una disminución en la fluidez de las asociaciones, puede ser útil hacer preguntas adicionales para identificar posibles conflictos internos como una fobia afectiva o disociativa: «¿Qué más te está ocurriendo ahora?» Esto puede ayudar a identificar la información relevante y la necesidad de una nueva intervención. También puede ayudar al paciente a tomar perspectiva y adoptar una postura metacognitiva. Dependiendo de la situación específica, hay diversas intervenciones posibles que pueden ayudar al paciente a recuperar la «postura plenamente consciente» que se necesita para un reprocesamiento eficaz. Algunos ejemplos incluyen: «no juzgues nada de lo que te venga», "tu propia rabia no ha sido la causa de los malos tratos que tu ... (el agresor) te ha causado con el abuso emocional», "irealmente necesitas esta emoción ahora?» (a veces los pacientes creen que necesitan aferrarse a una vieja emoción), «permítete sentir todas tus sensaciones» $\mathrm{O}$ «déjalo salir . . . está bien».

La centralidad de una alianza terapéutica adecuada. A lo largo de la fase de desensibilización, los clínicos deben estar atentos a los momentos de falta de sintonía y problemas relacionales. Como se ha señalado anteriormente, el factor más central para apoyar el reprocesamiento estructurado para los pacientes con TLP es un terapeuta centrado, calmado, firme, que sea un apoyo y que tenga una relación terapéutica suficientemente fuerte con el paciente. Desde este punto de partida, hay diversas intervenciones técnicas que pueden ser muy útiles, pero estas mismas intervenciones también pueden ser completamente ineficaces en ausencia de una alianza terapéutica adecuada.

Finalización de la sesión. Los principios y pasos procedimentales estándar de EMDR para el reprocesamiento con personas con TEPT, sin TLP ni DESNOS, se orientan a la consecución de una sesión completa. Sin embargo, cuando se ofrece reprocesamiento a personas con TLP, es mejor hacer un buen trabajo, aunque sea poco, y acabar con una sesión incompleta, que 
empujar para tratar de completar el reprocesamiento de una diana específica (Leeds y Mosquera, 2012). De hecho, cuando se trabaja con pacientes con TLP, a menudo es prudente o necesario cerrar las sesiones cuando técnicamente están incompletas. Cuando las sesiones quedan incompletas, los terapeutas pueden considerar una serie de pasos hacia el cierre. Estos incluyen preguntar por el nivel de activación emocional y somática del paciente; intervenciones de estabilización, enraizamiento y presentificación; análisis de la sesión con énfasis en la "mentalización»; breve instalación de declaraciones metacognitivas que reflejen los beneficios; y sugerencias de tareas o pruebas de autocuidado.

Una instalación parcial y temprana que facilite notar el cambio y la mentalización. Después de asegurar que el paciente se siente bien y está orientado, siente control sobre sus emociones y es capaz de reflexionar sobre la sesión, el terapeuta puede preguntar sobre la sensación del paciente de haber sacado algún beneficio, revelación o realización de la sesión. A continuación, el terapeuta puede considerar «instalar» brevemente cualquier logro o realización que haya sucedido durante la sesión o cualquier recurso necesario que pueda ayudar al paciente a afrontar el recuerdo. Estas instalaciones breves solo implican habitualmente dos o tres tandas de EBL, y no se debería presionar para obtener una validez de la cognición de 7. La declaración que refleja el logro o realización debe, por supuesto, ser realista. Por ejemplo, si el paciente solicita instalar la afirmación que prefiere, "que esto no ha sucedido", no sería aceptable. Sin embargo, el terapeuta puede proponer una declaración de posibilidad alternativa más adaptativa: «Algún día superaré esto . . . algún día esto no me hará daño», o cualquier otra posibilidad saludable que el paciente pueda creer realmente en ese momento.

\section{Reevaluación}

La mayoría de las dianas solo se reprocesarán hasta el final después de varias sesiones. Durante la fase de reevaluación, con la que se abren las sesiones posteriores, es esencial "comprobar el trabajo realizado", indagando sobre las experiencias del paciente después de la sesión anterior, así como preguntando por la diana concreta de la sesión anterior. Al decidir cómo proceder, es importante no perder el foco y es esencial evitar "saltar de diana en diana» (Mosquera, 2012b). El paciente puede traer a cada sesión diferentes problemas para trabajar, pero es importante encontrar un equilibrio entre reconocer las preocupaciones actuales mientras se mantiene el foco y seguir el rumbo marcado con dianas bien seleccionadas (Leeds y Mosquera, 2012). Es importante tener en mente un plan terapéutico claro pero flexible, ya que estos pacientes a menudo funcionan de manera muy caótica.

\section{Conclusiones}

EMDR es una terapia prometedora para los pacientes con TLP, pero su aplicación requiere varias adaptaciones a los procedimientos estándar de EMDR que fueron desarrollados originalmente para casos más simples de trastorno de estrés postraumático. El subgrupo de pacientes con TLP que presentan características más prominentes de disociación estructural se abordará mejor con intervenciones para afrontar la disociación estructural (González y Mosquera, 2012). La experiencia clínica sugiere que muchos pacientes con TLP solo necesitan cantidades limitadas de intervención de estabilización y ligeras modificaciones en los procedimientos estándar de EMDR para acceder al material traumático y procesarlo con seguridad, tales como las descritas en este artículo. Un aspecto central en el desarrollo y la organización de los planes de tratamiento EMDR seguros y eficaces para pacientes con TLP, es el desarrollo de habilidades conceptuales, de percepción, de procedimiento, e intersubjetivas que precisan los terapeutas para desarrollar el trabajo en cada una de las ocho fases de EMDR. El desarrollo de estas habilidades requiere educación avanzada especializada, formación y supervisión. Casos publicados anteriormente (Brown y Shapiro, 2006; Wesselmann y Potter, 2009) y una de las futuras publicaciones de series de casos (Mosquera, González y Leeds, 2014) documentan resultados positivos de la aplicación de EMDR como se describe en este artículo. Creemos que está justificado realizar investigaciones adicionales para examinar los principios que se han enumerado en este resumen. Por ejemplo, según estos principios, los sujetos seleccionados por cumplir los criterios del TLP podrían asignarse al azar a un número similar de sesiones de tratamiento con DBT o con terapia EMDR.

\section{Referencias bibliográficas}

Adler-Tapia, R. y Settle, C. (2009). Evidence of the efficacy of EMDR with children and adolescents in individual psychotherapy: A review of the research published in peerreviewedjournals. Journal of EMDR Practiceand Research,3(4), 232-247. http: / / dx.doi.org / 10.1891/1933-3196.3.4.232

Agrawal, H. R., Gunderson, J., Holmes, B. M. y Lyons-Ruth, K. (2004). Attachment studies with borderline patients: A review. Harvard Review of Psychiatry, 12(2), 94-104. 
Bakermans-Kranenburg, M. J., van Uzendoorn, M. H., Bokhorst, C. L. y Schuengel, C. (2004). The importance of shared environment in infant-father attachment: A behavioral genetic study of the attachment q-sort. Journal of Family Psychology, 18(3), 545-549.

Ball, J. S. y Links, P. S. (2009). Borderline personality disorder and childhood trauma: Evidence for a causal relationship. Current Psychiatry Reports, 11(1), 63-68.

Barone, L., Fossati, A. y Guiducci, V. (2011). Attachment mental states and inferred pathways of development in borderline personality disorder: A study using the adult attachment interview. Attachment y Human Development, 13(5), 451-469. http://dx.doi.org/10.1080/ 14616734.2011 .602245

Bateman, A. y Fonagy, P. (2004). Psychotherapy for borderline personality disorder: Mentalization-based treatment. Oxford, United Kingdom: Oxford University Press.

Battle, C. L., Shea, M. T., Johnson, D. M., Yen, S., Zlotnick, C., Zanarini, M. C., . . . Morey, L. C. (2004). Childhood maltreatment associated with adult personality disorders: Findings from the Collaborative Longitudinal Personality Disorders Study. Journal of Personality Disorders, 18, 193-211.

Bisson, J. y Andrew, M. (2007). Psychological treatment of post-traumatic stress disorder (PTSD). Cochrane Database of Systematic Reviews, 18(3), CD003388. http:// dx.doi.org/10.1002/14651858.CD003388.pub3

Bokhorst, C. L., Bakermans-Kranenburg, M. J., Fearon, R. M. P., Van IJzendoorn, M. H., Fonagy, P. y Schuengel, C. (2003). The importance of shared environment in mother-infant attachment security: A behavioral genetic study. Child Development, 74(6), 1769-1782.

Briere, J. (2004). Inventory of Altered Self-Capacities. Lutz, Florida: Psychological Assessment Resources.

Brown, S. y Shapiro, F. (2006). EMDR in the treatment of borderline personality disorder. Clinical Case Studies, 5(5), 403-420.

Brussoni, M. J., Jang, K. L., Livesley, W. J. y MacBeth, T. M. (2000). Genetic and environmental influences on adult attachment styles. Personal Relationships, 7, 283-289.

Chu, J. A. y Dill, D. L. (1991). Dissociation, borderline personality disorder, and childhood trauma. The American Journal of Psychiatry, 148, 812-813.

Classen, C., Pain, C., Field, N. y Woods, P. (2006). Posttraumatic personality disorder: A reformulation of the complex posttraumatic stress disorder and borderline personality disorder. Psychiatric Clinics of North America, 29, 87-112.

Cohen, P., Crawford, T. N., Johnson, J. G. y Kasen, S. (2005). The children in the community study of developmental course of personality disorder. Journal of Personality Disorders, 19, 466-486.

Collins, A. M. y Loftus, E. F. (1975). A spreading activation theory of semantic processing. Psychological Bulletin, 82, 407-428.

Courtois, C. A., Ford, J. D. y Cloitre, M. (2009). Best practices in psychotherapy for adults. En C. A. Courtois y J. D.
Ford (Eds.), Treating complex traumatic stress disorders: An evidence based guide (pp. 82-103). New York, NY: Guilford Press.

Crawford, T. N., Livesley, W. J., Jang, K. L., Shaver, P. R., Cohen, P. y Ganiban, J. (2007). Insecure attachment and personality disorder: A twin study of adults. European Journal of Personality, 21, 191-208.

Dalenberg, C. J. (2000). Countertransference and the treatment of trauma. Washington, DC: American Psychological Association.

Driessen, M., Beblo, T., Reddemann, L., Rau, H., Lange, W., Silva, A., . . Ratzka, S. (2002). Is the borderline personality disorder a complex post-traumatic stress disorder?The state of research. Nervenarzt, 73(9), 820-829.

Dworkin, M. (2005). EMDR and the relational imperative: The therapeutic relationship in EMDR treatment. New York, NY: Routledge.

Field, A. y Cottrell, D. (2011). Eye movement desensitization and reprocessing as a therapeutic intervention for traumatized children and adolescents: A systematic review of the evidence for family therapists. Journal of Family Therapy, 33(4), 374-388. http://dx.doi .org/10.1111/j .1467-6427.2011.00548.x

Foa, E. B., Keane, T. M., Friedman, M. J. y Cohen, J. A. (2009). Effective treatments for PTSD: Practice guidelines from the International Society for Traumatic Stress Studies. New York, NY: Guilford Press.

Fonagy, P., Gergely, G., Jurist, E. L. y Target, M. (2002). Affect regulation, mentalization, and the development of the self. New York, NY: Other Press.

Galletly, C. (1997). Borderline-dissociation comorbidity. American Journal of Psychiatry, 154, 1629.

Gelinas, D. J. (2003). Integrating EMDR into phase-oriented treatment for trauma. Journal of Trauma and Dissociation, 4(3), 91-135.

Gianoli, M. O., Jane, J. S., O’Brien, E. y Ralevski, E. (2012). Treatment for comorbid borderline personality disorder and alcohol use disorders: A review of the evidence and future recommendations. Experimental and Clinical Psychopharmacology, 20(4), 333. http:/ / dx.doi.org/10 .1037/ a0027999

Goldsmith, H. H. y Harman, C. (1994). Temperament and attachment: Individuals and relationships. Current Directions in Psychological Science, 3, 53-57.

Golier, J., Yehuda, R., Bierer, L., Mitropoulou, V., New, A., Schmeidler, J., . . S Siever, J. (2003). The relationship of borderline personality disorder to posttraumatic stress disorder and traumatic events. American Journal of Psychiatry, 160, 2018-2024.

Gonzalez, A. y Mosquera, D. (2012). EMDR and dissociation: The progressive approach. Charleston, SC: Amazon Imprint. Gonzalez, M., Mosquera, D. y Leeds, A. (2011). Family experiences in childhood scale. Retrieved from http: / / www.intra-tp.org

Goodman, M. y Yehuda, R. (2002). The relationship between psychological trauma and borderline personality disorder. Psychiatric Annals, 33, 337-345. 
Grover, K. E., Carpenter, L. L., Price, L. H., Gagne, G. G., Mello, A. F., Mello, M. F. y Tyrka, A. R. (2007). The relationship between childhood abuse and adult personality disorder symptoms. Journal of Personality Disorders, 21(4), 442-447.

Gunderson, J. G. y Sabo, A. (1993). The phenomenological and conceptual interface between borderline personality disorder and post-traumatic stress disorder. American Journal of Psychiatry, 150, 19-27.

Harter, S. (1999). The construction of the self: A developmental perspective. New York, NY: Guilford Press.

Hase, M. (2010). CravEx: An EMDR approach to treat substance abuse and addiction. En M. Luber (Ed.), Eye movement desensitization and reprocessing (EMDR) scripted protocols: Special populations (pp. 467-488). New York, NY: Springer Publishing.

Hase, M., Schallmayer, S. y Sack, M. (2008). EMDR reprocessing of the addiction memory: Pretreatment, posttreatment, and 1-month follow-up. Journal of EMDR Practice and Research, 2(3), 170-179. http:/ / dx.doi.org/ 10.1891/1933-3196.2.3.170

Herman, J. L. (1992). Complex PTSD: A syndrome in survivors of prolonged and repeated trauma. Journal of Traumatic Stress, 5(3), 377-391.

Hesse, E. (1999). The adult attachment interview: Historical and current perspectives. En J. Cassidy y P. R. Shaver (Eds.), Handbook of attachment: Theory, research and clinical applications (pp. 395-433). New York, NY: Guilford Press.

Hill, A. B. (1965). The environment and disease: Association or causation? Proceedings of the Royal Society of Medicine, 58, 295-300.

Hofmann, A. (2004). EMDR in the treatment of complex PTSD. Paper presented at the EMDR International Association Conference, Montreal, Quebec.

Hofmann, A. (2010). The inverted EMDR standard protocol for unstable complex post-traumatic stress disorder. En M. Luber (Ed.), Eye movement desensitization and reprocessing (EMDR) scripted protocols: Special populations (pp. 313-328). New York, NY: Springer Publishing.

Horesh, N., Ratner, S., Laor, N. y Toren, P. (2008). A comparison of life events in adolescents with major depression, borderline personality disorder and matched controls: A pilot study. Psychopathology, 41(5), 300-306. http:// dx.doi.org/10.1159/000141925

Johnson, J. G., Cohen, P., Brown, J., Smailes, E. M. y Bernstein, D. P. (1999). Childhood maltreatment increases risk for personality disorders during early adulthood. Archives of General Psychiatry, 56, 600-606.

Kernberg, O. F. (1993). Severe personality disorders: Psychotherapeutic strategies. New Haven, CT: Yale University Press.

Knipe, J. (1999). Targeting defensive avoidance and dissociated numbing. EMDRIA Newsletter, 4(2), 10, 25.

Knipe, J. (2003). “It was a golden time ... .”: Treating narcissistic vulnerability. En P. Manfield (Ed.), EMDR casebook (pp. 296-319). New York, NY: Norton.
Knipe, J. (2005). Targeting positive affect to clear the pain of unrequited love, codependence, avoidance, and procrastination. En R. Shapiro (Ed.), EMDR solutions: Pathways to healing (pp. 189-213). New York, NY: Norton.

Knipe, J. (2009). "Shame is my safe place": AIP targeting of shame as a psychological defense. En R. Shapiro (Ed.), EMDR solution II: For depression, eating disorders, performance, and more (pp. 49-89). New York, NY: Norton Professional Books.

Knipe, J. (2010a). Dysfunctional positive affect: Codependence or obsession with self-defeating behavior. En M. Luber (Ed.), Eye movement desensitization and reprocessing (EMDR) scripted protocols: Specialpopulations (pp. 463-465). New York, NY: Springer Publishing.

Knipe, J. (2010b). Dysfunctional positive affect: Procrastination. En M. Luber (Ed.), Eye movement desensitization and reprocessing (EMDR) scripted protocols: Special populations (pp. 453-458). New York, NY: Springer Publishing.

Knipe, J. (2010c). Dysfunctional positive affects: To assist clients with unwanted avoidance defenses. En M. Luber (Ed.), Eye movement desensitization and reprocessing (EMDR) scripted protocols: Special populations (pp. 451-452). New York, NY: Springer Publishing.

Knipe, J. (2010d). The method of constant installation of present orientation and safety [CIPOS]. En M. Luber (Ed.), Eye movement desensitization and reprocessing (EMDR) scripted protocols: Special populations (pp. 235-241). New York, NY: Springer Publishing.

Korn, D. L. (2009). EMDR and the treatment of complex PTSD: A review. Journal of EMDR Practice and Research, 3(4), 264-278. http:/ / dx.doi.org/10.1891/1933-3196.3.4.264

Korn, D. L. y Leeds, A. M. (2002). Preliminary evidence of efficacy for EMDR resource development and installation in the stabilization phase of treatment of complex posttraumatic stress disorder. Journal of Clinical Psychology, 58(12), 1465-1487. http://dx.doi.org/10.1002/ jclp.10099

Korzekwa, M. I., Dell, P. F. y Pain, C. (2009). Dissociation and borderline personality disorder: An update for clinicians. Current Psychiatry Reports, 11(1), 82-88.

Laporte, L. y Guttman, H. (1996). Traumatic childhood experiences as risk factors for borderline and other personality disorders. Journal of Personality Disorders, 10, 247-259.

Leeds, A. M. (1998). Lifting the burden of shame: Using EMDR resource installation to resolve a therapeutic impasse. En P. Manfield (Ed.), Extending EMDR, A case book of innovative applications (pp. 256-282). New York, NY: Norton.

Leeds, A. M. (2006, September). Learning to feel good about positive emotions with the positive affect tolerance and integration protocol. Paper presented at the annual meeting of the 11th EMDR International Association, Philadelphia, PA.

Leeds, A. M. (2009). A guide to the standard EMDR protocols for clinicians, supervisors, and consultants. New York, NY: Springer Publishing. 
Leeds, A. M. (2012, November). Defense and Affect Restructuring in EMDR. Taller presentado para EMDR España, Madrid.

Leeds, A. M. y Mosquera, D. (2012, October). Borderline Personality Disorder and EMDR. Taller presentado en la conferencia EMDRIA de 2012, "Attachment: Healing Developmental Trauma,” Washington, DC.

Lieb, K., Völlm, B., Rücker, G., Timmer, A. y Stoffers, J. M. (2010). Pharmacotherapy for borderline personality disorder: Cochrane systematic review of randomised trials. The British Journal of Psychiatry, 196(1), 4-12. http:/ / dx.doi.org/10.1192/bjp.bp.108.062984

Linehan, M. M. (1993). Skills training manual for treating borderline personality disorder. New York, NY: Guilford Press.

Litt, B. (2007). The child as identified patient: Integrating contextual therapy and EMDR. En F. Shapiro, F. W. Kaslow y L. Maxfield (Eds.), Handbook of EMDR and family therapy processes (pp. 306-324). Hoboken, NJ: Wiley.

McCullough, L. (1997). Changing character: Short-term anxiety-regulating psychotherapy for restructuring defenses, affects, and attachment. New York, NY: Basic Books.

McCullough, L. (2003). Treating affect phobia: A manual for short-term dynamic psychotherapy. New York, NY: Guilford Press.

McLean, L. M. y Gallop, R. (2003). Implications of childhood sexual abuse for adult borderline personality disorder and complex posttraumatic stress disorder. The American Journal of Psychiatry, 160, 369-371.

Mosquera, D. (2004). Diamantes en Bruto II. Programa psicoeducativo para personas con Trastorno Límite de la Personalidad. Madrid, Spain: Ediciones Pléyades.

Mosquera, D. (2012a, April). Installing self-care patterns in TLP. A crucial step for achieving stabilization. Taller presentado en el Aula Complutense de la Personalidad, Madrid.

Mosquera, D. (2012b, March). Treating borderline personality disorder with EMDR. Taller presentado en la conferencia Nacional de EMDR en Holanda.

Mosquera, D. y Gonzalez, A. (2011a, June). Personality disorders and EMDR. Taller presentado en 12 Conferencia de EMDR Europa en Viena, Austria.

Mosquera, D. y Gonzalez, A. (2011b). Personality disorders, relational trauma and EMDR. Taller presentado en la conferencia Nacional de EMDR en Roma, Italia.

Mosquera, D., Gonzalez, A. y Leeds, A. (2014). EMDR therapy for borderline personality disorder: A case series. Journal of EMDR Practice and Research. Manuscrito presentado para su publicación.

Mosquera, M., Gonzalez, A. y van der Hart, O. (2011). Borderline personality disorder, childhood trauma and structural dissociation of the personality. Revista Persona, 11 (Suppl. 1), 1-73.

Najavits, L. (2002). The Guilford substance abuse series: Seeking safety: A treatment manual for PTSD and substance abuse. New York, NY: Guilford Press.

Najavits, L. M., Weiss, R. D., Shaw, S. R. y Muenz, L. R. (1998). "Seeking Safety": Outcome of a new cognitive behavioral psychotherapy for women with posttraumatic stress disorder and substance dependence. Journal of Traumatic Stress, 11, 437-456.

O'Connor, T. G. y Croft, C. M. (2001). A twin study of attachment in preschool children. Child Development, 72, 1501-1511.

Pagura, J., Stein, M. B., Bolton, J. M., Cox, B. J., Grant, B. y Sareen, J. (2010). Comorbidity of borderline. Journal of Psychiatric Research, 44(16), 1190-1198.

Paris, J. y Zweig-Frank, H. (1997). Dissociation in patients with borderline personality disorder. The American Journal of Psychiatry, 154, 137-138.

Paris, J. y Zweig-Frank, H. (2001). A 27-year follow-up of patients with borderline personality disorder. Comprehensive Psychiatry, 42, 482-487.

Paulsen, S. (1995). Eye movement desensitization and reprocessing: Its cautious use in the dissociative disorders. Dissociation, 8(1), 32-44.

Pearlman, L. A. y Courtois, C. A. (2005). Clinical applications of the attachment framework: Relational treatment of complex trauma. Journal of Traumatic Stress, 18(5), 449-459.

Pietrzak, R. H., Goldstein, R. B., Southwick, S. M. y Grant, B. F. (2011). Personality disorders associated with full and partial posttraumatic stress disorder in the U.S. population: Results from Wave 2 of the National Epidemiologic Survey on Alcohol and Related Conditions. Journal of Psychiatric Research, 45(5), 678-686.

Popky, A. J. (2005). DeTUR, an urge reduction protocol for addictions and dysfunctional behaviors. En R. Shapiro (Ed.), EMDR solutions: Pathways to healing (pp. 167-188). New York, NY: Norton.

Ross, C. A. (2007). Borderline personality disorder and dissociation. Journal of Trauma y Dissociation, 8(1), 71-80.

Roth, S., Newman, E., Pelcovitz, D., van der Kolk, B. y Mandel, F. S. (1997). Complex PTSD in victims exposed to sexual and physical abuse: Results from the DSMIV eld trial for posttraumatic stress disorder. Journal of Traumatic Stress, 10, 539-556.

Sabo, A. N. (1997). Etiological significance of associations between childhood trauma and borderline personality disorder: Conceptual and clinical implications. Journal of Personality Disorders, 11, 50-70.

Shapiro, F. (1989). Eye movement desensitization: A new treatment for post-traumatic stress disorder. Journal of Behavior Therapy and Experimental Psychiatry, 20, 211-217.

Shapiro, F. (2001). Eye movement desensitization and reprocessing, basic principles, protocols and procedures. New York, NY: Guilford Press.

Shapiro, F. (2007). EMDR, adaptive information processing, and case conceptualization. Journal of EMDR Practice and Research, 1(2), 68-87. http:/ / dx.doi .org/10.1891/19333196.1.2.68

Shapiro, F. (2010, March). Mente humana, psicoterapia y EMDR. Paper presented at the XVII Scienti c Symposium "From Neurobiology to Nosology of Mental Disorders,” Lilly Foundation, Madrid, Spain. 
Siever, L. J., Torgersen, S., Gunderson, J. G., Livesley, W. J. y Kendler, K. S. (2002). The borderline diagnosis III: Identifying endophenotypes for genetic studies. Biological Psychiatry, 51(12), 964-968.

Timmerman, I. G. y Emmelkamp, P. M. (2001). The relationship between traumatic experiences, dissociation, and borderline personality pathology among male forensic patients and prisoners. Journal of Personality Disorders, 15(2), 136-149.

Trull, T. J., Sher, K. J., Minks-Brown, C., Durbin, J. y Burr, R. (2000). Borderline personality disorder and substance use disorders: A review and integration. Clinical Psychology Review, 20, 235-253.

Tyrka, A. R., Wyche, M. C., Kelly, M. M., Price, L. H. y Carpenter, L. L. (2009). Childhood maltreatment and adult personality disorder symptoms: Influence of maltreatment type. Psychiatry Research, 165(3), 281-287.

Ursano, R. J., Bell, C., Eth, S., Friedman, M., Norwood, A., Pfefferbaum, B., . . Y Yager, J. (2004). Practice guideline for the treatment of patients with acute stress disorder and posttraumatic stress disorder. American Journal of Psychiatry, 161(11 Suppl.), 3-31.

van der Hart, O., Brown, P. y van der Kolk, B. A. (1989). Pierre Janet's treatment of post-traumatic stress. Journal of Traumatic Stress, 2, 379-395.

van der Hart, O., Groenendijk, M., Gonzalez, A., Mosquera, D. y Solomon, R. (2013). Dissociation of the personality and EMDR therapy in complex trauma-related disorders: Applications in the stabilization phase. Journal of EMDR Practice and Research, 7(2), 81-94. http:/ dx.doi .org/10.1891/1933-3196.7.2.81

van der Hart, O., Nijenhuis, E. R. S. y Steele, K. (2006). The haunted self: Structural dissociation and the treatment of chronic traumatization. New York, NY: Norton.

van der Kolk, B., Roth, S., Pelcovitz, D., Sunday, S. y Spinazzola, J. (2005). Disorders of extreme stress: The empirical foundation of a complex adaptation to trauma. Journal of Traumatic Stress, 18(5), 389-399.

van der Kolk, B. A., Spinazzola, J., Blaustein, M. E., Hopper, J. W., Hopper, E. K., Korn, D. L. y Simpson, W. B. (2007). A randomized clinical trial of eye movement desensitization and reprocessing (EMDR), fluoxetine, and pill placebo in the treatment of posttraumatic stress disorder: Treatment effects and long-term maintenance. Journal of Clinical Psychiatry, 68(1), 37-46.
Watkins, J. G. (1971). The affect bridge: A hypnoanalytic technique. Journal of Clinical and Experimental Hypnosis, 19(1), 21-27.

Watkins, J. G. (1990). Watkins' affect or somatic bridge. En D. C. Hammond (Ed.), Handbook of hypnotic suggestions and metaphor (pp. 523-524). New York, NY: Norton.

Wesselmann, D. y Potter, A. E. (2009). Change in adult attachment status following treatment with EMDR: Three case studies. Journal of EMDR Practice and Research, 3(3), 178-191. http: / / dx.doi .org/10.1891/1933-3196.3.3.178

Yen, S., Shea, M. T., Battle, C. L., Johnson, D. M., Zlotnick, C., Dolan-Sewell, R., . . . McGlashan, T. H. (2002). Traumatic exposure and posttraumatic stress disorder in borderline, schizotypal, avoidant, and obsessive-compulsive personality disorders: Findings from the collaborative longitudinal personality disorders study. The Journal of Nervous and Mental Disease, 190, 510-518.

Young, J. E., Zangwill, W. M. y Behary, W. E. (2002). Combining EMDR and schema-focused therapy: The whole may be greater than the sum of the parts. En F. Shapiro (Ed.), EMDR and the paradigm prism (pp. 181-208). Washington, DC: American Psychological Association Press.

Zanarini, M. C. (2000). Childhood experiences associated with the development of borderline personality disorder. Psychiatric Clinics of North America, 23, 89-101.

Zanarini, M. C., Frankenburg, F. R., Hennen, J., Reich, D. B. y Silk, K. R. (2004). Axis I comorbidity in patients with borderline personality disorder: 6-year follow-up and prediction of time to remission. The American Journal of Psychiatry, 161, 2108-2114.

Zanarini, M. C., Yong, L., Frankenburg, F. R., Hennen, J., Reich, D. B., Marino, M. F. y Vujanovic, A. A. (2002). Severity of reported childhood sexual abuse and its relationship to severity of borderline psychopathology and psychosocial impairment among borderline inpatients. Journal of Nervous and Mental Disease, 190, 381-387.

Zimmerman, M. y Mattia, J. I. (1999). Axis I diagnostic comorbidity and borderline personality disorder. Comprehensive Psychiatry, 40, 245-252.

La correspondencia sobre este artículo se ha de dirigir a Dolores Mosquera, INTRA-TP, Instituto para el estudio del Trauma y los Trastornos de la Personalidad, General Sanjurjo 111, $5^{\circ}$ 15006, A Coruña, España. E-mail: doloresmosquera@gmail.com 